\title{
Entrelacs
}

Cinéma et audiovisuel

\section{De victime à hors-la-loi : l'émancipation par la transgression dans Baise-moi et Thelma \& Louise}

Jacinthe Dupuis

\section{(2) OpenEdition}

\section{Journals}

Édition électronique

URL : http://journals.openedition.org/entrelacs/351

DOI : 10.4000/entrelacs.351

ISSN : 2261-5482

Éditeur

Éditions Téraèdre

Référence électronique

Jacinthe Dupuis, « De victime à hors-la-loi : l'émancipation par la transgression dans Baise-moi et Thelma \& Louise », Entrelacs [En ligne], 9 | 2012, mis en ligne le 25 octobre 2012, consulté le 19 avril 2019. URL : http://journals.openedition.org/entrelacs/351 ; DOI : 10.4000/entrelacs.351

Ce document a été généré automatiquement le 19 avril 2019

Tous droits réservés 


\title{
De victime à hors-la-loi : l'émancipation par la transgression dans Baise-moi et Thelma \& Louise
}

\author{
Jacinthe Dupuis
}

1 Dans le film Thelma \& Louise de Ridley Scott comme dans Baise-moi de Virginie Despentes, les personnages féminins, après avoir été victimes de viol, commettent un meurtre et partent en cavale pour fuir les forces de l'ordre. Dans ces deux films, des road movies qui n'en sont pas réellement, la femme hors-la-loi représente une femme qui n'en est plus réellement une. Dans les deux cas, la transgression de la loi est figurée par un meurtre celui du violeur chez Thelma \& Louise et celui du frère de Manu dans Baise-moi, mais surtout, elle correspond à une transgression de genre pour les protagonistes. Sitôt les personnages de l'autre côté de la loi, sitôt les codes de la représentation du genre transformés : le féminin émancipé de la loi du père devient insaisissable, irrécupérable. Toutefois, ces femmes doublement hors-la-loi sont aussi doublement mises en échec. En quittant la loi du père qui constitue la structure du pouvoir organisé, elles se retrouvent face au chaos de l'anarchie, au vide (à la mort) qui les attend à la fin de la route. En analysant conjointement Baise-Moi et Thelma \& Louise, nous démontrerons que la position de hors-la-loi dans laquelle se placent les héroïnes est également une manière de transgresser et de subvertir les lois du genre sexuel telles que prescrites par le patriarcat.

2 Il n'est pas de réaction comparable à celle qu'a engendrée la sortie du film Baise-Moi de Virginie Despentes et Coralie Trinh Thi, sur les écrans français en 2000. Premier film à être banni en France en vingt-huit ans, Baise-moi, accusé de violence gratuite et de pornographie, ne sera finalement projeté sur les écrans qu'après une méticuleuse censure et une classification X. À l'aube du $21^{\mathrm{e}}$ siècle, Despentes avait-elle vraiment à apprendre quelque chose aux autres, après des films majeurs sur le sujet tels que Clockwork Orange (Stanley Kubrick, 1971) et Natural Born Killer (Oliver Stone, 1994), qui constituent euxmêmes une réflexion sur la violence que l'on dit gratuite?

3 Si le film est malgré tout sorti en salle en 2000, ce n'est pas sans de nombreuses protestations et demandes de censure. À ce sujet, deux aspects méritent d'être soulignés. 
La majorité des demandes de censure, telle que celles de la $\mathrm{BBFC}^{1}$ et de la $\mathrm{OFBC}^{2}$, exigeaient le retrait des scènes de viol, sous prétexte que:«violent pornography may excite aggressive responses from some male viewers" (MacKenzie, 2002, p. 315). Paradoxalement, la BBFC traduit le titre équivoque de Baise-moi par « Rape me » (violemoi), une phrase sans ambiguïté qui fragilise la notion de consentement et renforce l'idée que la femme est « une créature rendue responsable du désir qu'elle suscite » (Despentes, 2006 : 53). Le côté narguant et provocateur, l'appel au défi sensé émaner du titre est évacué. Ailleurs, le film a été plus adéquatement traduit par Fuck me, qui renferme la même évocation de sexualité et d'insulte que le titre original. En refusant de montrer à l'écran une scène de viol et en modifiant un titre qui constituaient eux-mêmes une critique du pouvoir, la BBFC touche au nœud du problème sur le discours dominant sur le viol : « [On] nous faire savoir qu'il n'y a rien de plus grave, et en même temps, qu'on ne doit ni se défendre, ni se venger » (Despentes, $2006: 49$ ).

Dans le film de Despentes comme dans celui de Ridley Scott, le viol constitue non seulement l'élément déclencheur de l'action mais l'aspect central d'une critique sociale sur l'inégalité des rapports de genre/sexe. En effet, dans la mise en scène des deux films, la notion de loi est placée en parallèle à celles des codes du genre par l'entremise des figures de hors-la-loi que deviennent Thelma, Louise, Nadine et Manu. La transgression, de la loi comme du genre, se fait dans les mêmes conditions, c'est-à-dire dans la violence et à contre-courant.

\section{Tué violable}

5 À quinze minutes de la scène finale de Thelma \& Louise, Thelma s'interroge sur la fidélité de sa partenaire. Lorsque Louise la rassure, Thelma lui explique : «Something's crossed over in me. I can't go back. I just couldn't live ». Pour le personnage, revenir en arrière, rebrousser chemin signifie revenir dans son passé de femme soumise et "violée ". Maintenant qu'elle est passée de l'autre côté de la loi, qu'elle s'est dégagée du patriarcat, elle se sent vivante et «awake »(terme dans le scénario qui s'oppose à «sedate», l'état dans lequel elle disait se sentir avec son mari Darryl). Cette phrase est prononcée après plusieurs jours de cavale, en plein désert, dans une scène qui anticipe la scène finale où les deux femmes font leur "ultime traversée » en plongeant volontairement avec leur voiture dans le Grand Canyon, sous le regard stupéfait d'une vingtaine de policiers armés. Elle renvoie également aux premières scènes du film qui précèdent le viol, et à ce qui ne devait être qu'un week-end entre copines, scènes qui mettent en relief la situation d'oppression dans laquelle se trouvent les protagonistes. Dans la scène initiale, on voit en alternance les deux femmes qui se parlent au téléphone, Thelma en robe de chambre dans sa cuisine et Louise en uniforme dans le restaurant où elle travaille. Les deux personnages endossent des rôles féminins traditionnels et se trouvent dans des décors passablement chargés, sonoriquement pour Louise et visuellement pour Thelma. La contrainte de l'espace dans lequel la femme est « enfermée » est mise en relief par l'aquarium sur lequel Louise s'attarde, montrant des poissons à la fois prisonniers et constamment observés. Leur brève conversation est supervisée par des figures de pouvoir, le patron de Louise et le mari de Thelma. Finalement, comme si les normes de la féminité étaient pour elle un poids, Thelma entame son voyage (originalement de 2 jours) avec plusieurs valises remplies de vêtements, qui disparaîtront complètement au fil de l'histoire, puisque le 
personnage finira par être simplement vêtu d'un jeans et d'un t-shirt noir, sur lequel on voit une tête de mort qui semble sourire et l'inscription Drivin my life away.

6 Si la figure de hors-la-loi féminine dérange, c'est parce qu'elle incarne une femme pour qui aucune règle ne s'applique, et qu'elle porte atteinte à la cohérence de l'hétéronormativité. Elle n'est donc plus tout à fait une femme, c'est-à-dire celle qui se définit par rapport à l'homme. Dans les deux œuvres, ce n'est pas un hasard si cette figure se construit par l'entremise d'une juxtaposition entre un viol et un meurtre, marques du refus des protagonistEs de se faire assigner les caractéristiques du genre féminin. Dans son article sur le viol et la loi, "Rape: On Coercion and Consent", Catharine A. MacKinnon écrit : « To be rapable, a position that is social not biological, defines what a woman is " (MacKinnon dans Conboy, 1998: 49). De façon semblable, dans King Kong théorie, Virginie Despentes attribue son sentiment d'impuissance au moment du viol dont elle a été la victime quand elle était jeune adulte, à son sexe : «C'est le projet du viol qui refaisait de moi une femme (Despentes, 2006: 51)». Le viol est une entreprise de réassignation de rôle, une "représentation crue et directe du pouvoir» qui place la femme en position horizontale, qui la subordonne, la condamne à subir. Le viol que la femme porte en elle en fait une victime éternellement potentielle, comme si elle était essentiellement privée d'agentivité et de subjectivité. Par conséquent, le refus du viol dans ces conditions ne peut qu'entraîner la mort de l'homme en tant que personne ayant un accès illimité à la sexualité féminine. Il constitue également ce que Sharon Marcus appelle une réécriture du scénario du viol (the rape script) parce qu'il déplace le sens $d u$ viol de ce qu'il promeut (la violence contre la femme) au geste d'exclusion qu'il comprend: "We can begin to develop a feminist discourse on rape by displacing the emphasis on what the rape script promotes - male violence against women - and putting into place what the rape script stultufies and excludes - women's will, agency, and capacity for violence » (Marcus dans Butler et al., 1992 : 395). Ce déplacement du sens, qui correspond au refus absolu des conditions de possibilité du patriarcat et des rôles sexuels qui lui sont inhérents, constitue l'acte de transgression ultime qui met en marche la cavale (le déplacement physique) vers le Mexique dans Thelma \& Louise et vers les Vosges pour Nadine et Manu, comme si les héroïnes étaient à la recherche d'un autre lieu où les lois (du genre et pénales) ne pèseraient pas sur elles.

7 Ces deux actions (viol et meurtre) constituent le point pivot des deux narrations, le moment où la femme se redresse et, au sens propre comme au sens figuré, prend la parole et le contrôle. Dans le cas de Thelma \& Louise, pendant la scène de viol, alors que Harlan maintient Thelma couchée sur la voiture, Louise, bien droite, brandit le fusil (apporté par Thelma au début du voyage) contre lui. Les deux protagonistes sont à armes égales - un homme contre un pistolet. Mais Louise ne tire pas, elle parle: «Looks like you've got a real fucked up idea of fun...In the future, when a women cries like this, she ain't havin' any fun ». Ainsi, ce n'est pas tant le viol qui déclenche le coup de fusil que l'insistance de l'homme: devant les femmes qui s'éloignent, il persiste à les maintenir en position d'infériorité en leur lançant une injure qui rappelle encore une fois la supériorité phallique : «Suck my cock ». Le coup de fusil serait ici la réponse à cette injonction, la parole d'une femme dressée comme le violeur et ainsi, révolutionnaire. Comme Hélène Cixous l'indique: «Il est temps que la femme marque ses coups dans la langue écrite et orale... C'est... en relevant le défi du discours gouverné par le phallus, que la femme affirmera la femme autrement qu'à la place à elle réservée dans et par le symbole c'est-àdire le silence » (Cixous, 1976 : 43). Ainsi, la prise de parole, le Je féminin, est un outil de 
contre-pouvoir: « $\ldots$ only the writing $I$ has the potential to become the relovutionary $I$ since writing enlables woman to come into agency and to escape the confinement of objectification " (Smith, 1993: 165). De la femme qui se tient debout, "straight as an I", partira la balle, horizontalement mortelle, le prolongement de ses paroles et de son agentivité.

La transgression de la loi se produit de manière similaire dans Baise-moi. Lorsque le frère de Manu la soupçonne d'avoir été violée, il sort son pistolet, prêt à la vengeance. Devant l'impassibilité de sa sœur, il rétorque: "T'es même pas traumatisée. Putain tu me dégoûtes, salope ». Du coup, Manu le frappe, récupère l'arme, et tire sur son frère. Si le silence était pour Manu une solution de ne pas être une victime, il devient pour son frère le plaidoyer de culpabilité dont parle Despentes : «Puisque dans le viol, il faut toujours prouver qu'on n'était pas d'accord. La culpabilité est comme soumise à une attraction morale non énoncée, qui voudrait qu'elle penche toujours du côté de celle qui s'est fait mettre, plutôt que de celui qui a cogné " (Despentes, 2006: 48). Ne pas être traumatisé revient, dans le discours du frère, à être une salope, une affirmation à laquelle s'oppose Manu, qui brisera son silence d'un coup de feu.

9 Si le bruit du coup de feu représente la prise de parole symbolique qui brise simultanément le silence et la loi, le thème du viol dans les deux films joue également avec le principe de la loi du silence qui régit le viol, ou du moins qui gravite autour de lui et opprime les femmes. Comme si le viol était le lieu d'un vide sémantique, d'un trou, il est l'événement qu'on ne mentionne pas, qui ne "passe pas dans le symbolique" (Despentes, $2006: 43)$. Tout au long de Thelma \& Louise, des allusions a « what happened in Texas » sont lancées à Louise, mais ne trouvent jamais ancrage puisque Louise refuse d'en parler. Louise a refusé le statut de victime à travers le silence, parce que comme l'indique Sharon Marcus dans son article "Fighting Bodies, Fighting words", le rituel que constitue l'aveu dans la rémission d'une victime est une forme de cristallisation du viol comme inhérent au féminin: "It has become routine if not dogmatic, within the antisexual violence movement to assert that the telling of stories is crucial for re-establishing and re-configuring an assailed female subjectivity in which rape marks the limits of women's access to complete citizenship and self-possession » (Marcus dans Butler et al, 1992 : 395). Le silence ou le refus de l'aveu est ici une forme de préservation du non-sens que constitue le viol. Et lorsqu'elle pointe une arme sur l'homme qui viole Thelma, c'est pour que celle-ci échappe à ce qu'elle-même a dû supporter pour survivre sans devenir victime : le silence. Dans Baise-moi, Despentes choisit de mettre en valeur le décalage entre l'horreur du viol et le silence de la victime de manière simultanée, à travers la crudité des images graphiques de la scène du viol. Alors qu'on fait un gros plan sur la pénétration à la manière des films pornographiques, pour ensuite passer à l'image de Manu qui reçoit, silencieuse, les coups de rein de son assaillant, on maintient en arrièreplan, les cris désespérés de l'autre victime, dont la résistance ne fait qu'attiser la violence de son agresseur. Comme Louise dans le film de Scott, Manu contourne l'événement, le dédramatise : « ... c'est juste des trucs qui arrivent, on est jamais que des filles C'est rien à côté de ce qu'ils peuvent faire. C'est jamais qu'un coup de queue». Le discours (essentialiste) du viol est articulé de manière à « désautonomiser » la sexualité féminine, à enlever à la femme sa subjectivité : « the rape script [...] suggests that we view rape not as the invasion of the female inner space, but as the forced creation of female sexuality as a violated inner space. The horror of rape is not that it steals something from us but that it makes us into things to be taken " (Marcus dans Butler et al., 1992: 399). La suite des 
évènements montre que ce positionnement quant au rapport des femmes au viol n'est pas une résignation essentialiste, voulant que le viol soit inévitable. Celui-ci tend plutôt vers une réécriture du viol, une désacralisation du féminin et de son sexe.

\section{Jouer le jeu}

10 Une fois le loup-garou assassiné (l'homme séducteur devenu violeur), Thelma et Louise quittent le bar Silver bullet en vitesse et prennent la route en direction du Mexique. Manu, quant à elle, prend en otage Nadine, une escorte occasionnelle qui vient d'assassiner sa colocataire et qui deviendra son alliée. Thelma, Louise et Nadine ont brisé les lois du genre en refusant de devenir victime et ont enfreint la loi en commettant un meurtre. Dès lors, elles entrent dans un autre lieu: «This outlawry opens a space for a subversive representational practice, one in which categorizations of all sorts get deconstructed, but no norm is substituted in or privileged » (Russell, 2002, n. p.). C'est dans cet espace horsla-loi que les personnages transgressent les limites des représentations sexuelles: The ultimate project of Thelma and Louise is nothing less than the destruction of the taxonomies that sustain the law of the father/nation » (Russell, 2002, n. p.).

De l'autre côté de la loi, c'est-à-dire une fois le meurtre commis, le visage fermé et voilé de silence de la Manu-victime est remplacé par celui, défiant et rieur, de la femme horsla-loi. Ainsi, après avoir séduit et ramené à l'hôtel un homme peu attrayant, Manu, à genoux, s'esclaffe. Elle vient de vomir sur l'homme à qui elle faisait une fellation. De façon semblable, le visage de Thelma, maquillé et taché de sang par la main de Harlan, se transfigure au fil des plans, pour devenir poussiéreux et bronzé. Auparavant passif et apeuré, il se fait franc, provocateur et complice au moment où le personnage et sa partenaire font exploser l'énorme camion-citerne d'un homme qui les assénait de vulgarités. Dans cette scène, le rire est littéralement défigurant, au sens où il détruit l'image de la femme-objet que projette le camionneur. Alors que Louise tire dans les pneus, Thelma, dont le chandail arbore une tête de mort médusante qui semble elle aussi sourire, tire sur la remorque argentée, faisant exploser du même coup le reflet de femmeobjet. Les deux femmes éclatent de rire et reprennent la route, laissant derrière elles le camionneur, à genou devant le brasier. Si les personnages des deux films n'exercent pas de contrôle sur les lois du langage patriarcal, elles démontrent toutefois qu'elles peuvent contrôler de ce qui « sort de leur bouche ». Selon Sidonie Smith, le rire est un élément qui déplace le sens et dépolarise le pouvoir; c'est aussi un acte non-rationnel qui implique tout le corps :

12 "The effect of laughter on the body elides the gap between species and gestures toward the instability of boundaries separating one species from another, unhinging secure placements in hierarchie of meaning. It also breaks up the elegant, cool, controlled planes of statuesque representationalism, forcing the irrational through the lucid planes of reason and control.The sound itself breaks through the language of phallocentrism, a call from beyond, from the body, from elsewhere. Ultimately laughter breaks up the consolidation of a universalized, rational, unifying truth, destabilizing foundational notions of truth » (Smith, 1993: 167).

13 Dans les deux cas, l'homme est " désarmé » par cette perte de contrôle, par le glissement vers l'anarchique que provoque le rire. L'ouverture du visage et le rire qui fuse des bouches qu'on a auparavant fait taire, devient un geste politique de revendication et déplace le féminin à un autre niveau : «Literally, laughter breaks up the assembled and 
calm planes of the face; and as the movement of laughter breaks up the consolidated features of the face, laughter aligns the human with the animal, the grotesque body" (Smith, 1993 : 166). Le dispositif de séduction qu'elles mettent en place pour attirer une victime qu'elles rejettent et méprisent en riant par la suite, positionne les héroïnes à l'extérieur des lois et des conventions. Ainsi, le rire provoqué par la moquerie des héroïnes face à une attitude phallocrate est une sorte de revanche qui transgresse la loi du silence et qui agit comme contre-pouvoir en regard des codes du langage patriarcal auxquels les femmes sont généralement soumises.

Le langage des femmes hors-la-loi se situe du côté de l'anarchie parce qu'il ne relève pas de la dépolarisation du code patriarcal mais plutôt d'un lieu où les codes sont explosés. La frustration de l'homme souillé par le vomissement de Manu dans Baise-moi, et de l'homme au camion-citerne de Thelma \& Louise qui jure en regardant le ciel et crie «You bitches from hell ! » alors que son camion se consume, met en relief la perte du pouvoir moins au profit de la femme qu'au profit du chaos. Par le rire déstabilisant et irrationnel des horsla-loi, les actions langagières prennent une dimension performative. L'acte de parole qu'est le consentement est ce qui distingue une relation sexuelle d'un viol' ${ }^{3}$, et un viol qui s'accomplit prouve que cette parole est demeurée sans effet. Dans le cas de Baise-moi, la femme dont on a ignoré le refus de consentement se sert de sa bouche pour attaquer directement le masculin(en vomissant sur le sexe d'un homme dans le cas de Manu). En faisant exploser le camion-citerne du camionneur qui a refusé de retirer les paroles dégradantes qu'il venait de prononcer, Thelma donne une dimension matérielle à son acte de parole en attaquant la représentation du pouvoir masculin, la source de l'agression.

La hors-la-loi est une figure polymorphe qui n'obéit à aucune règle qu'on lui impose. Elle utilise des codes, tels ceux de la séduction, à des fins de vengeance. Sous ses allures félines (on pense à la tignasse ébouriffée et à l'ensemble au motif de léopard de Manu, à la position langoureuse de Thelma et Louise sur la décapotable alors que le camionneur s'approche d'elles la femme hors-la-loi devient vipère, un serpent du désert qui crache son venin après avoir ensorcelé sa victime. Elle ne compte pas arrêter sa cavale au bout de la route. Peut-être que l'abîme appelle réellement l'abîme, et que le trou laissé par le viol, « taillé dans le vif..., qui ne se referme jamais tout à fait » (Despentes, 2006 : 53), ne se comble réellement que par le grand trou final du suicide, de la mort choisie.

Bien que dans Baise-moi les deux héroïnes n'atteignent jamais leur but, puisque Manu est abattue dans une station-service et que Nadine est attrapée par les policiers au moment où elle a le fusil sur la tempe, celles-ci avaient soigneusement décidé comment mourir. Et si, dans Thelma \& Louise, c'est une fois confrontée aux forces policières que les deux femmes décident de plonger dans le grand Canyon, dans les deux films, le choix initial est le même : elles préfèrent «l'auto-exclusion à l'élimination sociale " (Fayard, 2006: 67). Pour Thelma et Louise, la mort n'est pas la fin, elle est la continuité de leur parcours: "Let's not get caught... Let's keep going ", dit Thelma en indiquant du regard le vide à sa partenaire. Elles scellent leur pacte d'un baiser et, devant la rangée d'armes à feu pointées sur elles, « they hit it » et volent dans le vide. Le plan séquence de la caméra suit la trajectoire de la voiture qui monte vers le ciel avant de plonger dans le trou du canyon.

Cet acte, accompli dans Thelma \& Louise et empêché dans Baise-moi, relève dans les deux cas d'une posture politique que prend la femme hors-la-loi : celle de se tenir debout devant la mort, devant sa propre mort. Contrairement à Manu et Nadine, qui avaient planifié leur suicide, Thelma et Louise ne mentionnent jamais l'acte. L'horizontalité, le 
passé et la rangée de policiers, c'est la mort, et celle-ci est déjà derrière elles. Et en écho à la question du policier qui a tenté de les sauver tout au long du film : «How many times are these women gonna get fucked? », le geste des deux femmes répond: une seule fois, elle ne se feront «baiser » qu'une seule fois. Ces dernières refusent de retourner dans la loi, celle qui les jugera, les punira et les soumettra. Elles ne se laisseront pas abattre, coucher par les balles des fusils pointés sur elles, et choisissent plutôt de s'envoler vers le ciel. Dans cette scène finale, l'élévation verticale de la voiture s'oppose, comme l'explique Jean-Luc Nancy dans son livre sur la levée du corps du Christ, Noli me Tangere, "à l'horizontalité du tombeau -ne le quittant pas, ne le réduisant pas à néant mais affirmant en lui la tenue (donc aussi la retenue) d'un intouchable, d'un inaccessible (Nancy, 2007 : p. 33) ». C'est ce que les deux hors-la-loi deviennent, intouchables, inatteignables, même dans leur grand saut final.

Construit de manière plus pessimiste, Baise-moi présente des personnages qui seront rattrapés par le système, la dernière scène du film montrant en plongée Nadine maintenue au sol par les policiers. Mais plusieurs indices montrent que cette fin n'est pas simplement la mise en échec des deux héroïnes par la société. Au moment où elles planifient leur suicide, Manu préfère le saut dans le vide " sans élastique » à l'immolation qu'elle trouve trop prétentieuse. Elle offre également à Nadine, qui se croit incapable de sauter, de la pousser dans le vide. Mais le saut n'aura jamais lieu puisque Manu est tuée prématurément en allant chercher un café. Nadine récupère le corps de son amie et l'amène sur le bord d'un lac, dépose un baiser sur ses lèvres et l'enveloppe dans une couverture avant d'y mettre le feu. Entre les gros plans sur fond noir du visage de Nadine, on entend le rire de Manu. À la scène finale, Nadine se retrouve elle aussi au bord de l'eau, tenant dans sa main l'arme, l'outil de son agentivité. L'image est entre-coupée de flashbacks de la scène où Nadine et Manu dansent ensemble. On y voit en plongée le visage de Manu, qui regarde Nadine au-dessus d'elle, un corps-à-corps qui passe par le regard. Sans Manu, Nadine ne peut sauter dans le vide. Et si se suicider est la continuité logique pour les deux hors-la-loi, rester en vie et se faire prendre revient à se laisser mourir. Le passage au noir que l'on voit après le gros plan de Nadine l'arme à la tempe est un coup de feu qui n'en est pas un, un non-coup de feu qui ramène le silence et qui est donc tout aussi assassin.

Dans ce qui apparaît être un cul-de-sac, pour le spectateur comme pour la police, Thelma et Louise choisissent de s'élancer. Il n'y a pas de cul-de-sac sur la route, comme il n'y a pas de fatalité dans le viol. Ce qui fait d'une femme une hors-la-loi n'est pas tant la transgression des normes du genre ou de l'identification sexuelle, que le refus d'être « toujours déjà violée ». Ce qui dérangeait tant dans le travail de Baise-moi c'est la création d'un féminin inviolable, chez qui la parole a autant de pouvoir que les gestes masculins. Chez Despentes comme chez Ridley Scott, la femme hors-la-loi est dangereuse parce qu'en refusant la possibilité du viol elle refuse l'essence même de ce qui crée la loi patriarcale. Par cette réécriture du discours du viol, qui ne se fait pas tant dans la violence que dans les mots, le pouvoir de l'homme et ses actes deviennent caducs, sans emprise ni effet. "Baise-moi » est un peu la phrase leitmotiv de ce revirement, à michemin entre la requête et l'insolence qui replace la femme inviolable en tant que sujet dans le jeu de la sexualité. 


\section{BIBLIOGRAPHIE}

\section{Filmographie}

DESPENTES, Virginie, et THRIN-THI, Coralie. Baise-moi, France, 2000, Canal +, 77 min.

SCOTT, Ridley. Thelma \& Louise, Etats-Unis, 1991, MGM, 130 min.

Bibliographie

BUTLER, Judith et Joan W. Scott, Feminists Theorise the Political, New York/Londres, Routhledge, 1992, $485 \mathrm{p}$.

CIXOUS, Hélène, « Le rire de la méduse », L'Arc, 61, 1975, p. 39-54

CONBOY, Kathy, MEDINA, Nadia, et STANBURY, Sarah, Writing on the Body. Female Embodiment and Feminist Theory, New York, Columbia University Press, 1998, 433 p.

DESPENTES, Virginie. King Kong théorie, Paris, Grasset, 2006, 159 p.

FAYARD, Nicole. « The rebellious body as parody: Baise-moi de Virginie Despentes », French Studies, vol. LX, 2006, No. 1, p. 63-77.

HORECK, Tania, Public Rape. Representing violation in fictions and film, New York/London, Routhledge, 2004, 195 p.

MacKENZIE, Scott, « Baise-moi, feminist cinemas and the censorship controversy », Screen, vol. 43, no 3, Automne 2002, p. 315-324.

NANCY, Jean-Luc. Noli me tangere, Paris, Bayard, 2003, 94 p.

RUSSELL, David. «I'm not gonna hurt you. Legal Penetration in Thelma and Louise », Americana: The Journal of American Popular Culture, Spring 2002, vol. 1, Issue 1 http:// www.americanpopularculture.com/journal/articles/spring_2002/russell.htm,

SMITH, Sidonie, Subjectivity, Identity and the Body, Bloomington and Indianapolis, Indiana University Press, 1993, 226 p.

\section{NOTES}

1. British Board of Film Classification (MacKenzie, 2002: 321)

2. Ontario Board of Film Classification (Canada) (MacKenzie, 2002: 320)

3. The law, speaking generally, defnies rape as intercourse with force or coercion and without consent. (MacKinnon dans Conboy, 1998: 41) 


\section{RÉSUMÉS}

Dans le film Thelma \& Louise de Ridley Scott comme dans Baise-moi de Virginie Despentes, on met en scène des personnages féminins qui, après avoir été victime de viol, commettent un meurtre et partent en cavale pour fuir les forces de l'ordre. Dans ces deux films, des road movies qui n'en sont pas réellement, la hors-la-loi féminine est la représentation d'une femme qui n'en est plus réellement une. L'article examine comment, dans ces deux films, la transgression de la loi au sens propre du terme, correspond également à une transgression des codes genre pour les héroïnes, codes qui notamment désignent le féminin comme être « violable ». Dans les deux oeuvres, sitôt les personnages de l'autre côté de la loi, sitôt les codes de la représentation du genre se transforment; le féminin émancipé de la loi du père devient insaisissable, irrécupérable et surtout, inviolable.

\section{AUTEUR}

\section{JACINTHE DUPUIS}

Est étudiante à la maîtrise en études littéraires à l'Université du Québec à Montréal, sous la direction de Martine Delvaux. Elle termine présentement son mémoire sur l'écriture de la sexualité féminine comme lieu de subversion du pouvoir patriarcal chez Alice Massat et Marie L. Elle a également collaboré à l'organisation de deux colloques internationaux, Insaisissables visages du féminin (2007) à Montréal et Femmes, création, politique (2008) à Cerisy. 